\title{
Throughput of Type II HARQ-OFDM/TDM Using MMSE-FDE in a Multipath Channel
}

\author{
Haris Gacanin and Fumiyuki Adachi \\ Department of Electrical and Communication Engineering, Graduate School of Engineering, Tohoku University, \\ Sendai, 980-8579, Japan \\ Correspondence should be addressed to Haris Gacanin, harisg@mobile.ecei.tohoku.ac.jp
}

Received 27 May 2009; Accepted 11 September 2009

Recommended by George Tsoulos

In type II hybrid ARQ (HARQ) schemes, the uncoded information bits are transmitted first, while the error correction parity bits are sent upon request. Consequently, frequency diversity cannot be exploited during the first transmission. In this paper, we present the use of OFDM/TDM with MMSE-FDE and type II HARQ to increase throughput of OFDM due to frequency diversity gain.

Copyright ( $) 2009$ H. Gacanin and F. Adachi. This is an open access article distributed under the Creative Commons Attribution License, which permits unrestricted use, distribution, and reproduction in any medium, provided the original work is properly cited.

\section{Introduction}

Packet services are dominant in future broadband wireless communications, where hybrid automatic repeat request (HARQ) is used for error control [1]. HARQ schemes combine error detection and forward error correction to make more efficient use of the channel. In type I HARQ, all the necessary parity bits are sent with each transmission. However, type II HARQ sends only the error detection parity bits, while the error correction parity bits are retransmitted upon request if the decoder detects errors. Therefore, with type II HARQ scheme a higher throughput can be achieved.

Wireless channel is characterized as a multipath propagation environment (i.e., frequency selective fading channel). Orthogonal frequency division multiplexing (OFDM) is attractive due to its robustness against multipath channel, while a drawback is its high peak-to-average power ratio (PAPR) [2]. OFDM with rate compatible punctured turbo (RCPT) coded HARQ [3] gives a good throughput. However, in type II HARQ-OFDM the uncoded packet is send first and consequently, neither coding nor frequency diversity gain is available. In essence, OFDM-based scheme (e.g., multicarrier CDMA) with type II HARQ [4] that efficiently exploits the channel frequency selectivity in the first transmission is preferable. Most of the work in this area preclude the power requirement of multicarrier signaling due to high PAPR.
In this paper, we present the use of OFDM combined with time division multiplexing (OFDM/TDM) using minimum mean square error frequency domain equalization (MMSE-FDE) [5] with type II HARQ to exploit the channel frequency selectivity. In type II HARQ OFDM/TDM during the first uncoded transmission the channel frequency selectivity is exploited through MMSE-FDE to increase the throughput of conventional OFDM with a lower PAPR.

The paper is organized as follows. Section 2 describes the system model. The performance is presented in Section 3. Section 4 concludes the letter.

\section{System Model}

The OFDM/TDM system model with HARQ is illustrated in Figure 1. At the transmitter, a cyclic redundancy check (CRC) coded sequence is input to the turbo encoder [6]. The parity sequences are punctured before transmission based on adopted HARQ scheme. The encoded sequences (i.e., a systematic (information) and two parity sequences) are stored in the buffer for transmissions.

We consider type II HARQ scheme represented by Systematic-Puncture period $P=x\left(S P_{x}\right)$. Parity sequences are punctured with $P=x$ and thus, $x$ different sequences of length $2 N / x$ are obtained, where $N$ is the CRC encoded 


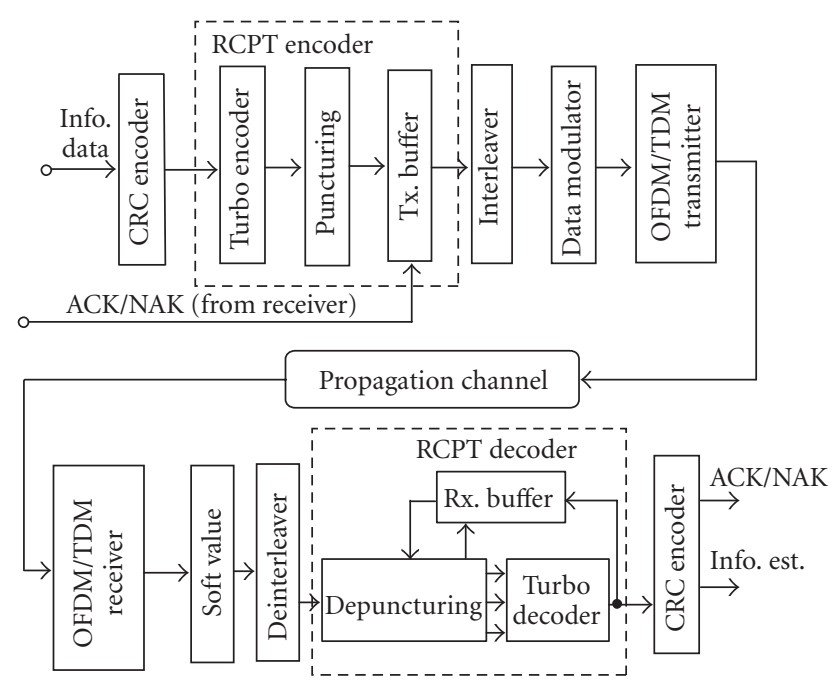

FIgURE 1: Transmission system model.

sequence length. For each puncturing period, the parity bit sequences are punctured such that the bits furthest apart in the two sequences are periodically selected. The puncturing matrices for $S P_{2}$ (binary notation) and $S P_{8}$ (octal notation) are given by [7]

$$
\begin{aligned}
S P_{2}= & \left(\begin{array}{ll}
1 & 1 \\
0 & 0 \\
0 & 0
\end{array}\right)\left(\begin{array}{ll}
0 & 0 \\
1 & 0 \\
0 & 1
\end{array}\right)\left(\begin{array}{ll}
0 & 0 \\
0 & 1 \\
1 & 0
\end{array}\right), \\
S P_{8}= & \left(\begin{array}{lll}
3 & 7 & 7 \\
0 & 0 & 0 \\
0 & 0 & 0
\end{array}\right)\left(\begin{array}{lll}
0 & 0 & 0 \\
2 & 0 & 0 \\
0 & 1 & 0
\end{array}\right)\left(\begin{array}{lll}
0 & 0 & 0 \\
0 & 0 & 2 \\
0 & 4 & 0
\end{array}\right) \\
& \left(\begin{array}{lll}
0 & 0 & 0 \\
0 & 2 & 0 \\
0 & 0 & 1
\end{array}\right)\left(\begin{array}{lll}
0 & 0 & 0 \\
0 & 0 & 4 \\
1 & 0 & 0
\end{array}\right)\left(\begin{array}{lll}
0 & 0 & 0 \\
0 & 1 & 0 \\
2 & 0 & 0
\end{array}\right) \\
& \left(\begin{array}{lll}
1 & 0 & 0 \\
0 & 0 & 0 \\
0 & 0 & 4
\end{array}\right)\left(\begin{array}{lll}
0 & 0 & 0 \\
0 & 0 & 1 \\
0 & 2 & 0
\end{array}\right)\left(\begin{array}{lll}
0 & 0 & 0 \\
0 & 4 & 0 \\
0 & 0 & 2
\end{array}\right) .
\end{aligned}
$$

In all the schemes the first (uncoded) transmission consists of only the systematic bits. The number of bits transmitted in the second transmission onwards differs depending on the puncturing period. The systematic bit sequence and punctured parity bit sequences are block interleaved and quadrature phase shift keying (QPSK) modulated.

After block interleaving, the interleaved bit sequences are fed to the OFDM/TDM modulator [5]. In OFDM/TDM, the inverse fast Fourier transform (IFFT) time window of conventional OFDM with $N_{c}$ subcarriers is divided into $K$ time slots (in this paper called OFDM/TDM frame) as illustrated in Figure 2 (the figure illustrates an example with $N_{c}=16$ and $K=4$, where $d(n)$ denotes the $n$th data modulated symbol within the each slot). Within each slot the OFDM signal with reduced number of subcarriers $N_{m}\left(=N_{c} / K\right)$ is sent. Thus, with OFDM/TDM scheme a lower PAPR is achieved in comparison with conventional OFDM. On the other hand, reducing the number of subcarriers will produce an increase in the out-of-band spectrum emission [8]. The guard interval (GI) is inserted over the entire frame with $K$ slots and the OFDM/TDM signal is sent over a multipath channel.

At the receiver, the GI is removed and $N_{c}$-point FFT is applied over the entire frame to decompose the received signal into its frequency components. One-tap MMSE-FDE is applied to take advantage of the channel frequency selectivity. The time-domain OFDM/TDM signal is recovered by $N_{c}$-point IFFT and then, OFDM demodulation is carried out by $N_{m}$-point FFT to obtain the decision variables for soft-value computation before channel decoding. Finally, CRC decoding is done to detect errors and if any errors are detected the transmission of parity bits is requested.

\section{Simulation Results}

We assume $N=1024, N_{c}=256, N_{g}=32$, and QPSK modulation. The channel is a 16-path block Rayleigh fading channel with power delay profile having decay factor $\beta$. The channel gains remain constant during one frame and vary frame by frame. The normalized Doppler frequency is assumed to be $f_{D} T_{s}=1 \times 10^{-3}$ (corresponds to a mobile terminal moving speed of about $50 \mathrm{~km} / \mathrm{h}$ for the $5 \mathrm{GHz}$ carrier frequency and the transmission data rate of $1 / T_{c}=$ $100 \mathrm{Msymbols} / \mathrm{s})$, where $1 / T_{s}=1 /\left[T_{c}\left(1+N_{g} / N_{c}\right)\right]$ is the transmission symbol rate. A rate $1 / 3$ turbo encoder with $(13,15)$ RSC component encoders having constraint length 4 and $S\left(=N^{1 / 2}\right)$-random internal interleaver and a $\log$-MAP decoder with 8 iterations are used. Before data modulation the encoded sequence is interleaved by block channel interleaver. We assume an ideal channel state information (CSI) (but channel estimation scheme for OFDM/TDM can be found in [9]). An error-free reverse channel and an ideal error detection are assumed. The maximum number of retransmissions is set to 100 .

Figure 3 shows the throughput as a function of the average bit energy-to-additive white gaussian noise (AWGN) power spectrum density ratio $E_{b} / N_{0}\left(=0.5 \times R \times\left(E_{s} / N_{0}\right) \times\right.$ $\left.\left(1+N_{g} / N_{c}\right)\right)$ with $K$ as a parameter for $\beta=0 \mathrm{~dB}$ (i.e., the strongest channel frequency selectivity) and QPSK modulation. The throughput in $\mathrm{bps} / \mathrm{Hz}$ is defined as the ratio of the number of information bits over the total number of transmitted bits, where the throughput loss due to the GI insertion is taken into consideration. As shown by Figure 3, the throughput of type II HARQ improves as $K$ increases because the FDE is designed to exploit the channel frequency selectivity, during the first uncoded transmission. The figure shows that the throughput in all cases approaches the value of about $1.8 \mathrm{bps} / \mathrm{Hz}$ due to GI insertion loss. When $E_{b} / N_{0}=$ $20 \mathrm{~dB}$ and $P=2$, throughput increases from 0.9 to about $1.1,1.4$, and 1.7 as $K$ increases from 1 (OFDM) to 4,16 , and 64, respectively. The throughput of OFDM/TDM slightly 


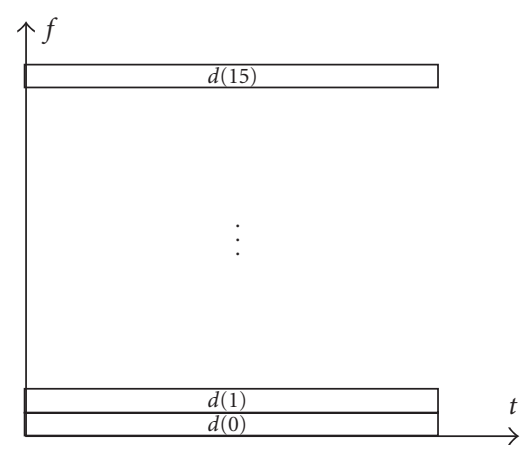

(a)

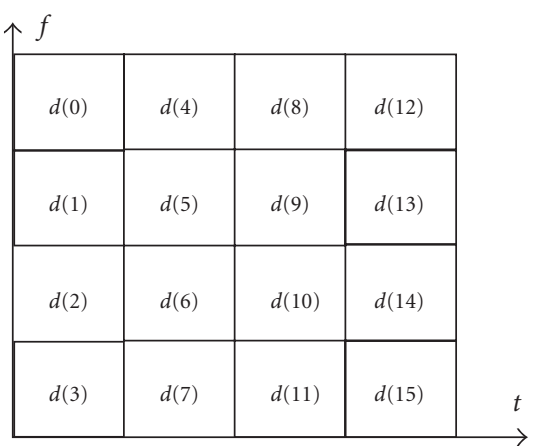

(b)

Figure 2: Conventional OFDM and OFDM/TDM frame structures.

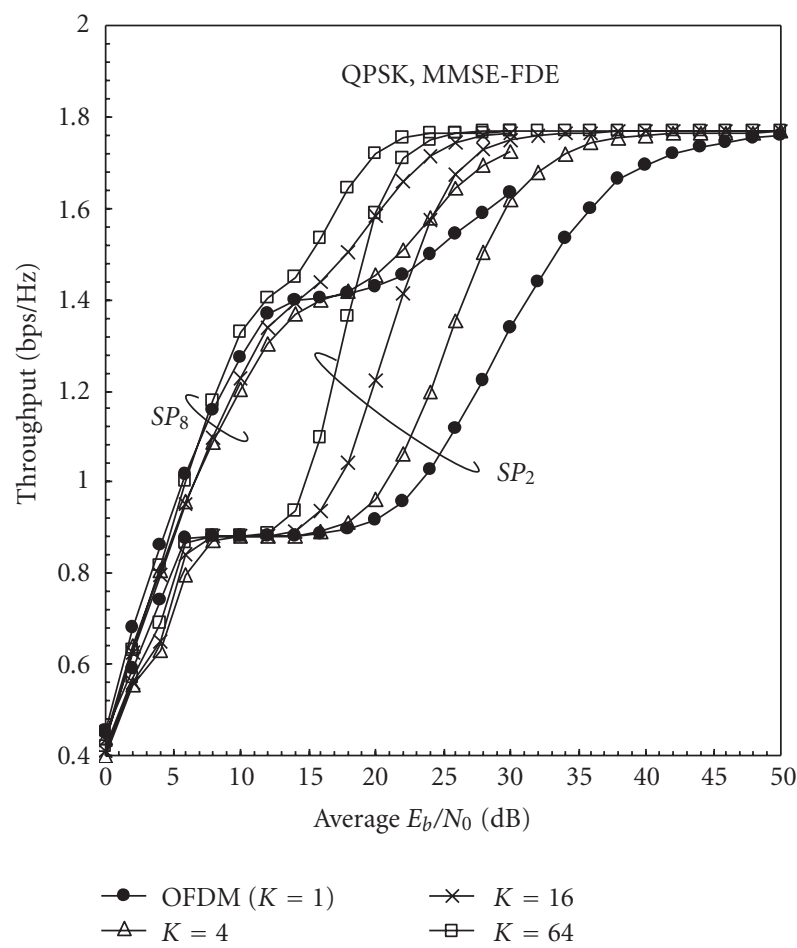

FIGURE 3: Throughput for different HARQ schemes: QPSK.

degrades in comparison with conventional OFDM $(K=1)$ in a lower $E_{b} / N_{0}$ region (e.g., $E_{b} / N_{0}<12 \mathrm{~dB}$ ); when $E_{b} / N_{0}=$ $5 \mathrm{~dB}$ and $P=2$, throughput decreases from 0.8 to about 0.7 as $K$ increases from 1 (OFDM) to 4 , respectively. This is because, in low $E_{b} / N_{0}$ region, always the transmission of parity bits is requested and error correction coding is carried out the number of subcarriers of OFDM/TDM is reduced in comparison with conventional OFDM and therefore, less frequency diversity gain through channel decoding is achieved by OFDM/TDM.

When $K$ increases, the residual interslot interference after MMSE-FDE will increase due to nonperfect compensation of the channel frequency selectivity, which may affect the higher-level modulation schemes. We illustrate in Figure 4

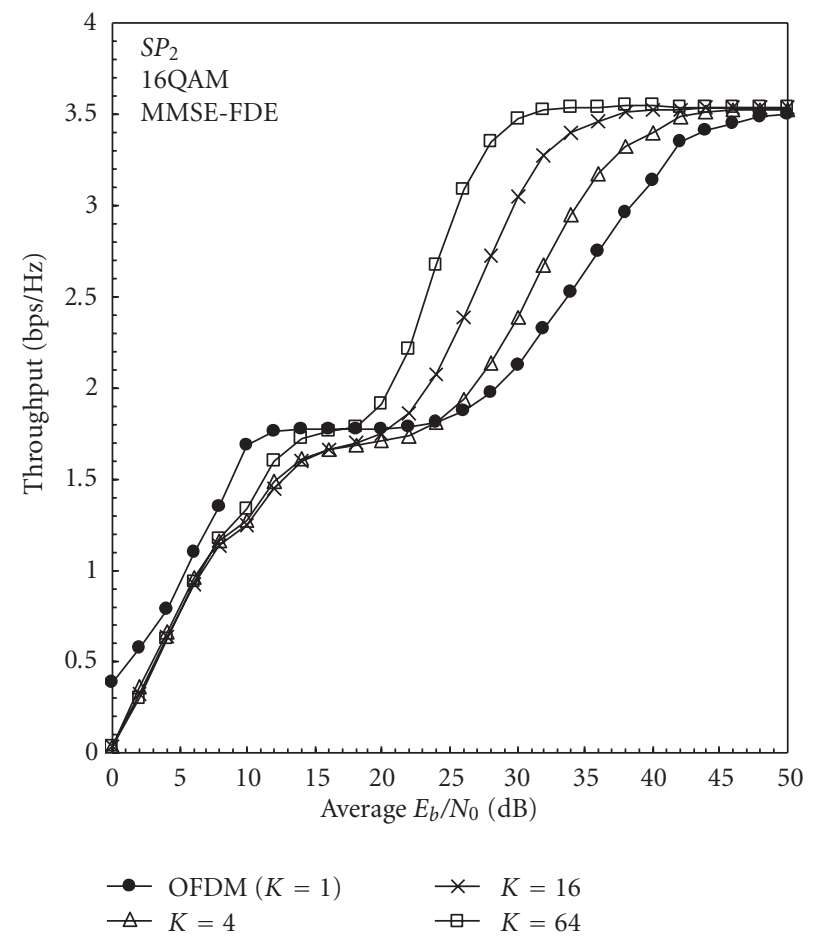

FIgURE 4: Throughput of OFDM/TDM with 16-QAM.

the throughput of OFDM/TDM using MMSE-FDE with $P=2$ in the case of 16-quadrature amplitude modulation (QAM). As shown by Figure 4, when $E_{b} / N_{0}>15 \mathrm{~dB}$, the throughput of OFDM/TDM improves as $K$ increases in comparison with conventional OFDM $(K=1)$. However, when $E_{b} / N_{0}<15 \mathrm{~dB}$, the throughput of OFDM/TDM with 16-QAM reduces in comparison with conventional OFDM $(K=1)$ due to the same reason stated earlier.

Dependency of the throughput on $\beta$ (i.e., the channel frequency-selectivity) with $K$ as a parameter is shown in Figure 5. The figure shows that, when $K=16$ and 64, the throughput of OFDM/TDM using MMSE-FDE reduces as $\beta$ increases, but again starts to increase after a certain level of $\beta$ is reached. The former is because lower frequency 


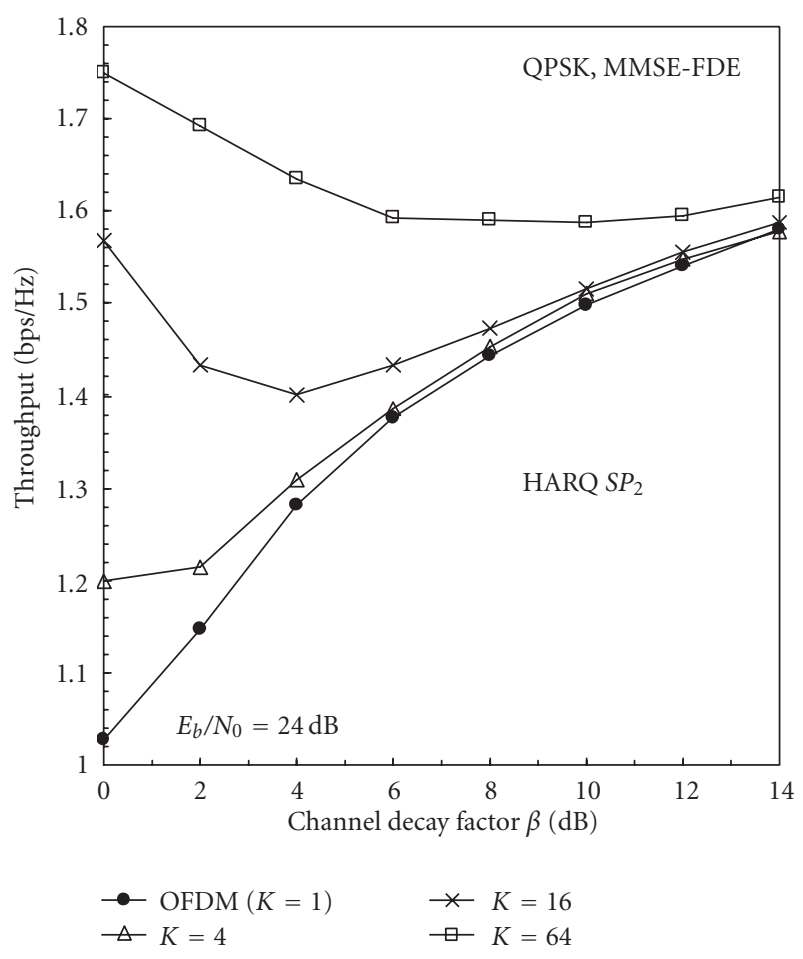

FIGURE 5: Impact of channel frequency selectivity: QPSK.

diversity gain is obtained as the channel becomes less frequency selective. The later is because, as $\beta$ increases, the signal spectrum over the entire bandwidth tends to drop simultaneously and the whole OFDM/TDM frame tends to be faded, resulting in bursty errors, which contribute to decrease the block error rate and hence, increase the throughput. However, it can be seen from the figure that OFDM/TDM achieves a higher throughput in comparison with conventional OFDM for lower $\beta$ (i.e., stronger channel frequency selectivity) due to frequency diversity gain during the first transmission.

\section{Conclusion}

In this paper, the use of OFDM/TDM with MMSE-FDE and type II HARQ was presented. It was shown that, during the first uncoded transmission, the throughput can be improved using OFDM/TDM with MMSE-FDE and type II HARQ due to the frequency diversity gain and lower PAPR in comparison with conventional OFDM. The best throughput is obtained with single carrier (SC)-FDE ( $K=256$ not shown here) [4], but OFDM-based scheme is preferable since a resource allocation (RA) [10] strategy may be applied to $\mathrm{OFDM} / \mathrm{TDM}$ to further enhance the performance. This is left as an interesting future study.

\section{Acknowledgment}

This work was supported by Grant-in-Aid for Scientific Research from Japan Society for the Promotion of Science.

\section{References}

[1] S. Lin and D. J. Costello, Error Control Coding: Fundamentals and Aplications, Prentice-Hall, Upper Saddle River, NJ, USA, 1983.

[2] S. Hara and R. Prasad, Multicarrier Techniques for 4G Mobile Communications, Artech House, Boston, Mass, USA, 2003.

[3] D. N. Rowitch and L. B. Milstein, "Rate compatible punctured turbo (RCPT) codes in a hybrid FEC/ARQ system," in Proceedings of the IEEE Global Telecommunications MiniConference (GLOBECOM '97), vol. 4, pp. 55-59, Phoenix, Ariz, USA, November 1997.

[4] S. Takaoka and F. Adachi, "HARQ with variable spreading factor for multicode MC-CDMA," Electronics Letters, vol. 42, no. 2, pp. 103-105, 2006.

[5] H. Gacanin, S. Takaoka, and F. Adachi, "Bit error rate analysis of OFDM/TDM with frequency-domain equalization," IEICE Transactions on Communications, vol. 89, no. 2, pp. 509-517, 2006.

[6] C. Berrou, A. Glavieux, and P. Thitimajshima, "Near optimum error correcting coding and decoding: turbo-codes," IEEE Transactions on Communications, vol. 44, no. 9, pp. 12611271, 1996.

[7] D. Garg and F. Adachi, "Rate compatible punctured turbocoded hybrid ARQ for OFDM in a frequency selective fading channel," in Proceedings of the IEEE Vehicular Technology Conference (VTC '03), vol. 57, pp. 2725-2729, Jeju, South Korea, April 2003.

[8] H. Gacanin and F. Adachi, "A comprehensive performance comparison of OFDM/TDM using MMSE-FDE and conventional OFDM," in Proceedings of the 67th IEEE Vehicular Technology Conference (VTC '08), pp. 1404-1408, Singapore, May 2008.

[9] H. Gacanin and F. Adachi, "On channel estimation for OFDM/TDM using MMSE-FDE in a fast fading channel," EURASIP Journal of Wireless Communications and Networking, vol. 2009, Article ID 481214, 9 pages, 2009.

[10] C. Y. Wong, R. S. Cheng, K. B. Letaief, and R. D. Murch, "Multiuser OFDM with adaptive subcarrier, bit, and power allocation," IEEE Journal on Selected Areas in Communications, vol. 17, no. 10, pp. 1747-1758, 1999. 

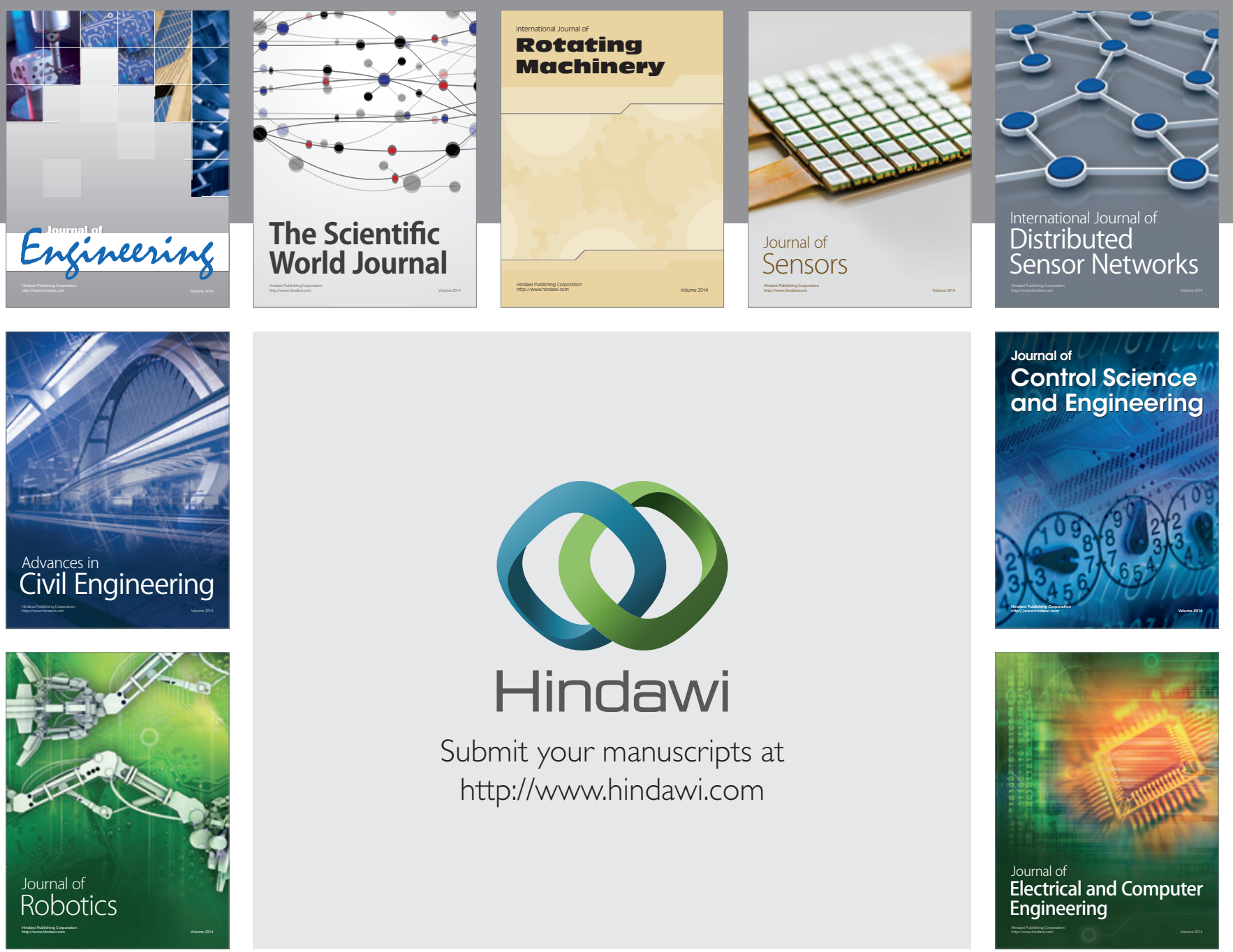

Submit your manuscripts at

http://www.hindawi.com
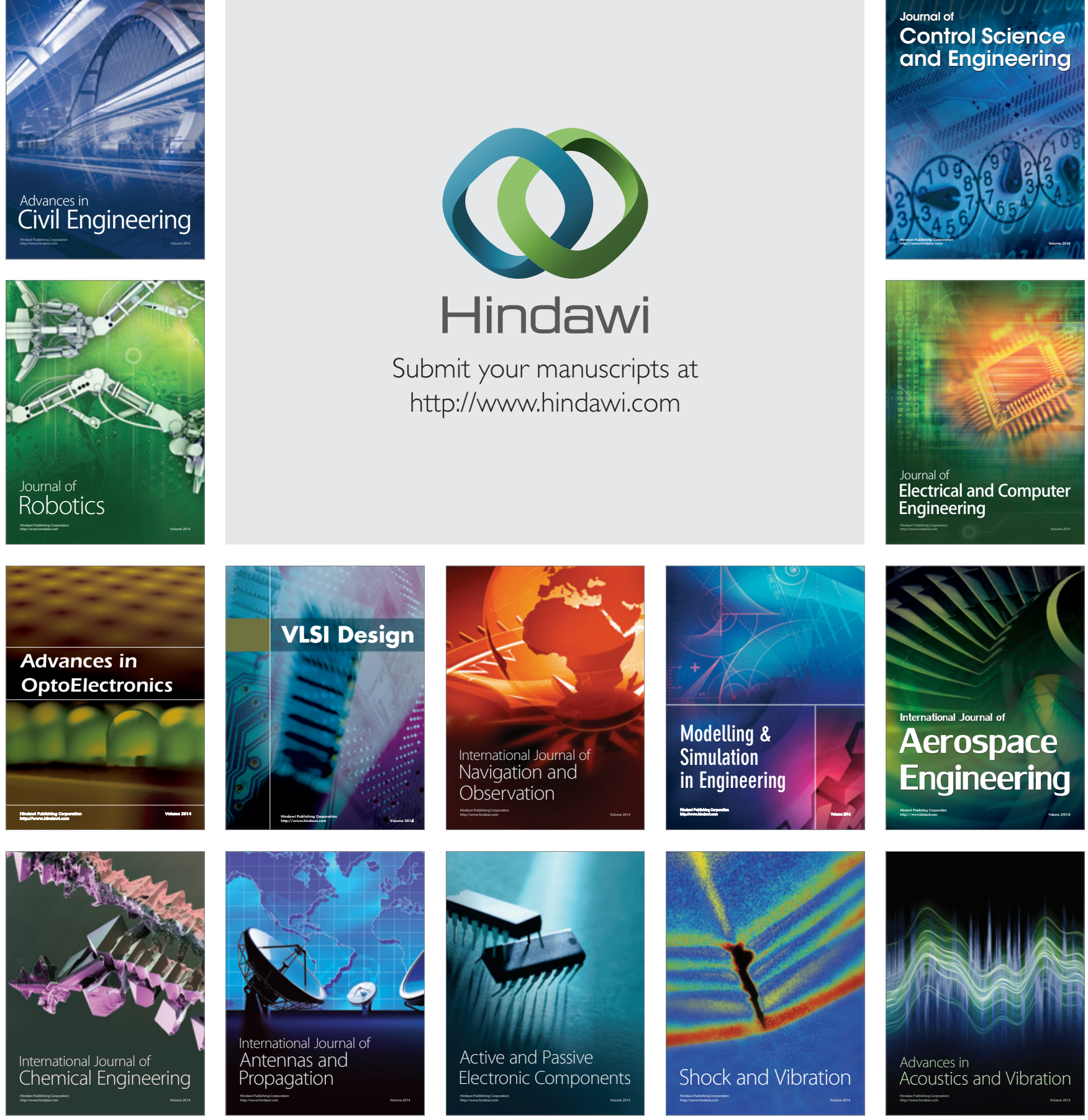